\title{
ORIGINAL ARTICLE \\ Thyroid hormone responsive QTL and the evolution of paedomorphic salamanders
}

\author{
SR Voss ${ }^{1}$, DK Kump ${ }^{1}$, JA Walker ${ }^{1}$, HB Shaffer ${ }^{2,4}$ and GJ Voss ${ }^{1,3}$ \\ The transformation of ancestral phenotypes into novel traits is poorly understood for many examples of evolutionary novelty. \\ Ancestrally, salamanders have a biphasic life cycle with an aquatic larval stage, a brief and pronounced metamorphosis, \\ followed by a terrestrial adult stage. Repeatedly during evolution, metamorphic timing has been delayed to exploit growth- \\ permissive environments, resulting in paedomorphic salamanders that retain larval traits as adults. We used thyroid hormone \\ (TH) to rescue metamorphic phenotypes in paedomorphic salamanders and then identified quantitative trait loci (QTL) for life \\ history traits that are associated with amphibian life cycle evolution: metamorphic timing and adult body size. We demonstrate \\ that paedomorphic tiger salamanders (Ambystoma tigrinum complex) carry alleles at three moderate effect QTL (met1-3) \\ that vary in responsiveness to TH and additively affect metamorphic timing. Salamanders that delay metamorphosis attain \\ significantly larger body sizes as adults and met2 explains a significant portion of this variation. Thus, substitution of alleles \\ at TH-responsive loci suggests an adaptive pleiotropic basis for two key life-history traits in amphibians: body size and \\ metamorphic timing. Our study demonstrates a likely pathway for the evolution of novel paedomorphic species from \\ metamorphic ancestors via selection of TH-response alleles that delay metamorphic timing and increase adult body size. \\ Heredity (2012) 109, 293-298; doi:10.1038/hdy.2012.41; published online 1 August 2012
}

Keywords: Ambystoma; paedomorphosis; evolution; QTL; thyroid hormone

\section{INTRODUCTION}

Many amphibians have life cycles with distinct larval and adult phases (Wilbur, 1980). The transition between phases, or metamorphosis, marks a period of development where larval traits are lost or remodeled and adult traits are gained. The timing of metamorphosis is critical because larval habitats present ecological growth opportunities for attaining body sizes and ages that affect survival and future reproduction in the adult phase. Theories predict that natural selection will favor delayed metamorphosis in growth-permissive habitats to increase adult body size, but early metamorphosis (and smaller body size) in less favorable habitats to reduce mortality risk (Wilbur and Collins, 1973; Gould, 1977; Werner, 1986). These predictions rest on genetic assumptions that have rarely been investigated in natural species. In general, little is known about the genetic architecture of amphibian life history traits and processes of genetic change that are associated with life cycle evolution.

Ancestrally, tiger salamanders (Shaffer and McKnight, 1996) have a biphasic life cycle with an aquatic larval stage, a brief and pronounced metamorphosis, followed by a terrestrial juvenile and adult stage. However, metamorphosis has been repeatedly altered during evolution, resulting in salamanders that retain larval traits into the adult, breeding stage (Shaffer, 1984; Shaffer and Voss, 1996). The retention of ancestral larval traits in the adult stage of derived forms is known as paedomorphosis and Ambystoma salamanders are the most widely known example of this important evolutionary phenomenon (Sprules, 1974; Gould, 1977). Whereas metamorphs are capable of terrestrial dispersal, obligatorily paedomorphic taxa are confined to isolated bodies of water. This suggests that metamorphic ancestors colonized stable aquatic habitats and that permissive ecological conditions selected for paedomorphic life histories. The fitness benefits of paedomorphosis include an earlier time to first reproduction, larger body size, larger clutch size, a higher probability of mating success (Semlitsch et al., 1988; Whiteman, 1994; Krenz and Sever, 1995; Ryan and Semlitsch, 1998) and more than one breeding event per year for some taxa.

Paedomorph expression in the Mexican axolotl (A. mexicanum) is associated with met1, a major effect quantitative trait locus (QTL) that regulates metamorphic timing (Voss and Shaffer, 1997, 2000; Voss and Smith, 2005). The met1 alleles from A. mexicanum delay metamorphic timing in comparison with alleles from metamorphic A. tigrinum tigrinum. From these results, Voss and Smith (2005) proposed a quantitative genetic model to explain the evolution of continuous metamorphic-timing phenotypes among tiger salamanders, as well as the origin of discontinuous, paedomorphic phenotypes. In habitats that allow extensions of the larval period, selection favors alleles that delay metamorphic timing because this is expected to increase adult body size, a component of fitness. In very stable aquatic habitats, selection favors alleles that permanently delay or

\footnotetext{
${ }^{1}$ Department of Biology and Spinal Cord and Brain Injury Research Center, University of Kentucky, Lexington, KY, USA; ${ }^{2}$ Department of Ecology and Evolution and Center for Population Biology, University of California, Davis, Davis, CA, USA and ${ }^{3}$ Math, Science, and Technology Center, Paul Laurence Dunbar High School, Lexington, KY, USA ${ }^{4}$ Current address: Department of Ecology and Evolutionary Biology, University of California, Los Angeles, CA 90095-1606, USA

Correspondence: Dr SR Voss, Department of Biology and Spinal Cord and Brain Injury Research Center, University of Kentucky, 101 TH Morgan Building, Lexington, KY 40506, USA.

E-mail: srvoss@uky.edu

Received 8 April 2012; revised 21 June 2012; accepted 25 June 2012; published online 1 August 2012
} 
block metamorphosis, allowing populations to capitalize on the reproductive benefits of the paedomorphic life history. Although paedomorphic phenotypes present extreme examples of metamorphic-timing evolution, their origin presumably traces to loci that coordinately regulate metamorphic timing and body size variation within biphasic life cycles.

Here, we present results from physiological and genetic experiments that support and extend Voss and Smith's (2005) model. Paedomorphosis and metamorphosis are associated with thyroid hormone (TH), the primary metamorphic hormone in amphibians and some fish (Laudet, 2011). During metamorphosis, TH activates transcriptional programs that cause regression of larval traits and development of adult traits. Paedomorphic species evolve via disruption of peripheral and central mechanisms of TH regulation (Shaffer and Voss, 1996). We used $\mathrm{TH}$ to induce ancestral metamorphic life history traits in paedomorphic species to reveal insights about the physiological and genetic basis of metamorphic timing. We show that three paedomorphic and one facultative salamander species complete metamorphosis in response to TH treatment at significantly different times, with the facultative species showing the quickest response and thus highest sensitivity to TH. We then show that met1 and two newly identified QTL (met2, met3) harbor alleles that vary in responsiveness to $\mathrm{TH}$ and, as a result, alter metamorphic timing. Salamanders that delay metamorphosis attain larger body sizes as adults and met2 explains a significant portion of this variation. Our results identify tissue response to $\mathrm{TH}$ as a genetically variable mechanism underlying a life history trade-off that is well known from ecological studies of amphibians with biphasic life cycles: the dependency of adult body size on metamorphic timing (Wilbur and Collins, 1973; Wilbur, 1980). Overall, our study supports the idea that paedomorphic tiger salamanders evolve from genetic changes of THresponsive loci that have adaptive pleiotropic effects on metamorphic timing and adult body size.

\section{MATERIALS AND METHODS}

\section{Genetic crosses, animal care and TH induction}

Eight adult salamanders from each of four paedomorphic species (A. andersoni, A. ordinarium, A. dumerilii and A. mexicanum) were collected under permit of the Mexican Government, and reared and induced with TH to undergo metamorphosis according to standard methods (Page et al., 2008). At approximately 120 days post fertilization (dpf), metamorphic ambystomatid species initiate metamorphosis and $50 \mathrm{~nm}$ T4 reliably induces metamorphosis in laboratory A. mexicanum. The rate and timing of metamorphosis was quantified by measuring gill length; gills are resorbed during metamorphosis. Subsequent to this, a female A. mexicanum/A. andersoni $\mathrm{F} 1$ hybrid was crossed to an A. mexicanum to generate approximately 300 AxAnl offspring. Larvae were reared individually in charcoal-filtered water and fed brine shrimp and California black worms (Lumbriculus variegatus); metamorphs were fed crickets and earthworms. At 90, 120, 300 and $400 \mathrm{dpf}$, individuals were anesthetized in $0.02 \%$ benzocaine and measured for morphometric traits: snout-vent length (distance from the snout to the posterior end of the cloaca), tail length (distance from the posterior end of the cloaca to the tail tip) and body weight. From $120 \mathrm{dpf}$ to metamorphic completion, rearing water contained $2.5 \mathrm{~nm}$ (T4; T2376; Sigma, St Louis, MO, USA) (15). Gender was determined by visual inspection of gonads or by cloacal size and shape. Methods for animal care and use were approved by University of California, Davis and University of Kentucky, Institutional Animal Care and Use Committees.

\section{Genetic linkage analysis}

Genotypes were obtained using Illumina Golden Gate and primer extension assays (Illumina, San Diego, CA, USA). Primer sequences, diagnostic polymorphisms and polymorphism detection assays are summarized in Supplementary File 1. Marker orders were determined using MultiPoint 2.2 (MultiQTL Ltd., Hafia, Israel) and the Kosambi (Kosambi, 1944) mapping function. QTL were identified using R/qtl (Broman et al., 2003) and the imputation method for quantitative traits (Sen and Churchill, 2001). As linear models showed that sex explained a significant amount of variation for tail length at $300 \mathrm{dpf}$ and body weight at $400 \mathrm{dpf}$, sex was included as an additive covariate in genome-wide scans for these traits. Genome-wide thresholds for evaluating the significance of QTL was determined from 1000 replicated data sets for each trait (Churchill and Doerge, 1994). These values ranged from log of odds ratio $(\mathrm{LOD})=2.71-2.83$ for probability of $\alpha=0.05$.

\section{RESULTS}

We collected paedomorphic salamanders from their natural habitats in Mexico, returned them to the lab, generated and reared offspring to $120 \mathrm{dpf}$, and tested the resulting larvae for metamorphic response to T4 $(50 \mathrm{~nm})$. Individuals of all four species resorbed their gills and underwent complete metamorphosis, but metamorphic timing differed significantly among the species (Figure 1). The shortest time to complete metamorphosis was observed for A. ordinarium, a species that facultatively metamorphoses in nature (Duellman, 1961). The other three species, A. andersoni, A. dumerilii and A. mexicanum, are obligate paedomorphs in their lacustrine natural habitats; these species responded more slowly to TH. These experiments demonstrate that independently derived paedomorphic species present different rates of $\mathrm{TH}$-induced metamorphosis in a common laboratory setting, and thus genetic variability in $\mathrm{TH}$ response.

To further investigate the genetic basis of $\mathrm{TH}$ response, we performed crosses to segregate 'paedomorphosis' alleles from two species, A. mexicanum and A. andersoni (Figure 2). An F1 female hybrid was crossed to an A. mexicanum male, and the backcross offspring (AxAn1) were reared to $120 \mathrm{dpf}$ and then subjected to a low-dose regime of T4 $(2.5 \mathrm{~nm})$ through $300 \mathrm{dpf}$ to examine subtle variation in T4 response. Approximately $85 \%$ of the AxAn 1 offspring survived and successfully resorbed their gills over a wide range of times (161-306 dpf). Only 1 of 30 control individuals that did not receive T4 metamorphosed naturally, whereas 8 individuals failed to undergo metamorphosis during the experiment. Those that completed metamorphosis included an approximately equivalent number of males

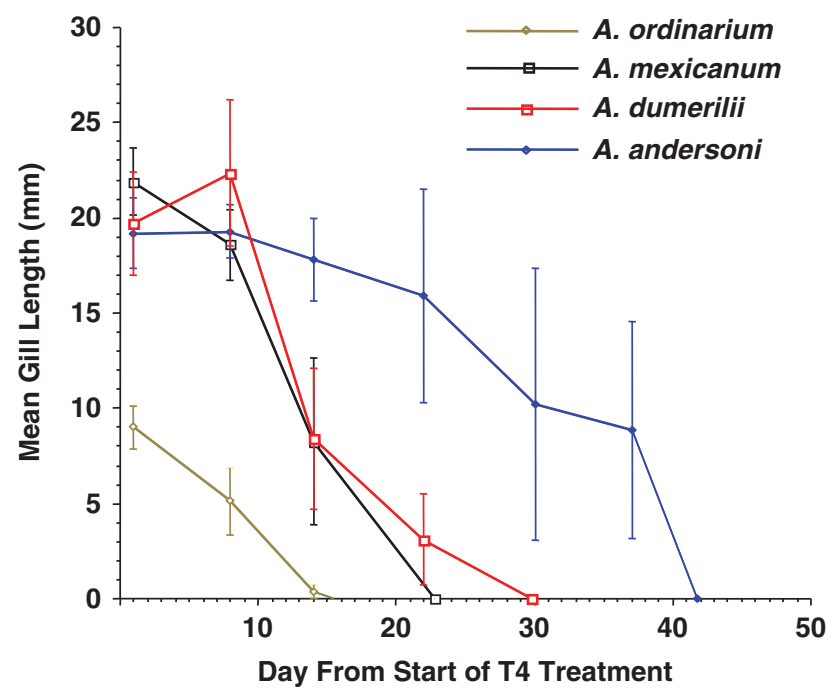

Figure 1 Plot showing pattern of gill resorption for T4-induced paedomorphic individuals from $A$. mexicanum, $A$. andersoni, $A$. ordinarium and $A$. dumerilii. Error bars are s.d. of means calculated from $N=8$ individuals per species. 

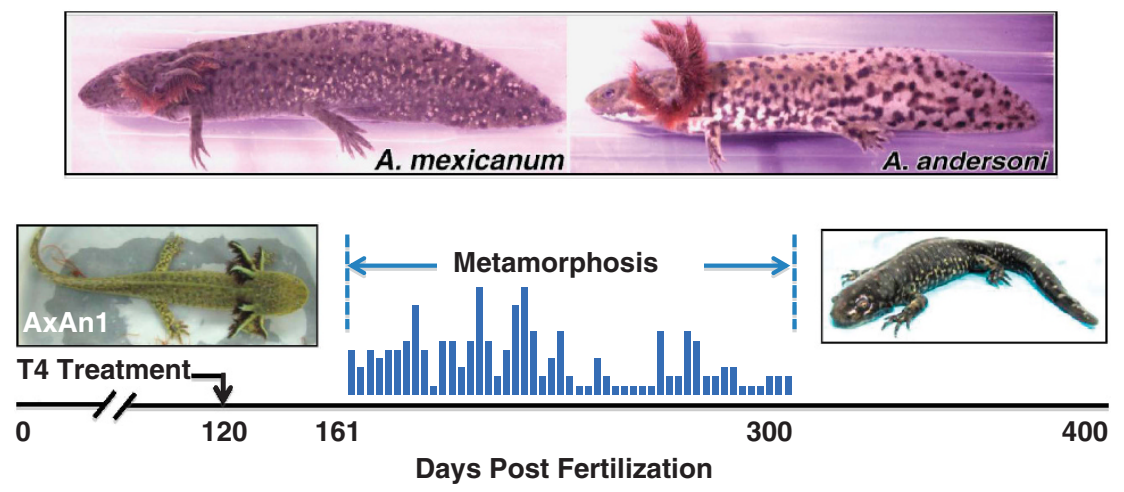

Figure 2 Representative A. mexicanum, A. andersoni and an F2 offspring deriving from a backcross of an F1 hybrid into A. mexicanum. The time line shows the timing of thyroid hormone treatment and the distribution of AxAn offspring metamorphic times.

( $N=124)$ and females $(N=130)$, and there were no gender-associated differences in metamorphic timing. However, metamorphic timing affected sexually dimorphic traits typical of naturally metamorphosing, terrestrial adult tiger salamanders (Howard et al., 1997). Individuals that delayed metamorphosis presented longer tails (a male-specific sign of sexual maturation) at $300 \mathrm{dpf}$ and were heavier at $400 \mathrm{dpf}$ (Figure 3). For the range of metamorphic times observed, males and females increased their adult body weight at $400 \mathrm{dpf}$ by approximately 0.12 and $0.14 \mathrm{~g}$, respectively, for each day that the metamorphic timing was delayed. Thus, age at metamorphosis varied inversely with adult morphological traits that are associated with fitness (body weight at a specific age), and the effect was approximately equivalent for dimorphic terrestrial male and female salamanders.

The correlations among metamorphic timing and adult fitness traits may be due to the same T4-sensitive loci with pleiotropic effects, or to independent loci. The difference is crucial, as it identifies the potential for these important life-history traits to evolve independently. We therefore created an AxAn1 genetic map by genotyping 185 molecular markers, the majority of which were used previously to map A. mexicanum $\times$ A. tigrinum linkage groups (Voss et al., 2011). Markers were spaced on average every $20 \mathrm{cM}$, although marker distribution was dense or sparse for some regions (Supplementary File 1). Genome-wide scans were performed to identify QTL for metamorphic timing, tail length at $300 \mathrm{dpf}$ and body weight at $400 \mathrm{dpf}$. Three significant LOD peaks were identified for metamorphic timing: two on linkage group (LG) 2 and one on LG7 (Figure 4). The two QTL from LG2 were separated by $136 \mathrm{cM}$, with the first marking the position of met1 $(c c d c 12: 270 \mathrm{cM})$ and the other identified at the position of nccrp1 near the end of LG2 (met2: $406 \mathrm{cM})$. The QTL (met3) on LG7 mapped near chmp2b. Altogether, the nearest markers defining these QTL explained $31 \%$ of the total variation in metamorphic timing, with A. mexicanum alleles at each locus having roughly equivalent (that is, each explained $10-11 \%$ total phenotypic variance) additive effects in delaying metamorphic timing. The met 1 allele from $A$. andersoni decreased average time to metamorphosis by approximately 19 days and thus had the same directional effect as the met 1 allele from metamorphic $A$. $t$. tigrinum, which decreased timing in mapping families with A. mexicanum on average by 36 days (Voss and Smith, 2005). These results demonstrate that met1-3 are differentially responsive to $\mathrm{T} 4$ and genetically variable among independently evolved, paedomorphic ambystomatid salamanders.
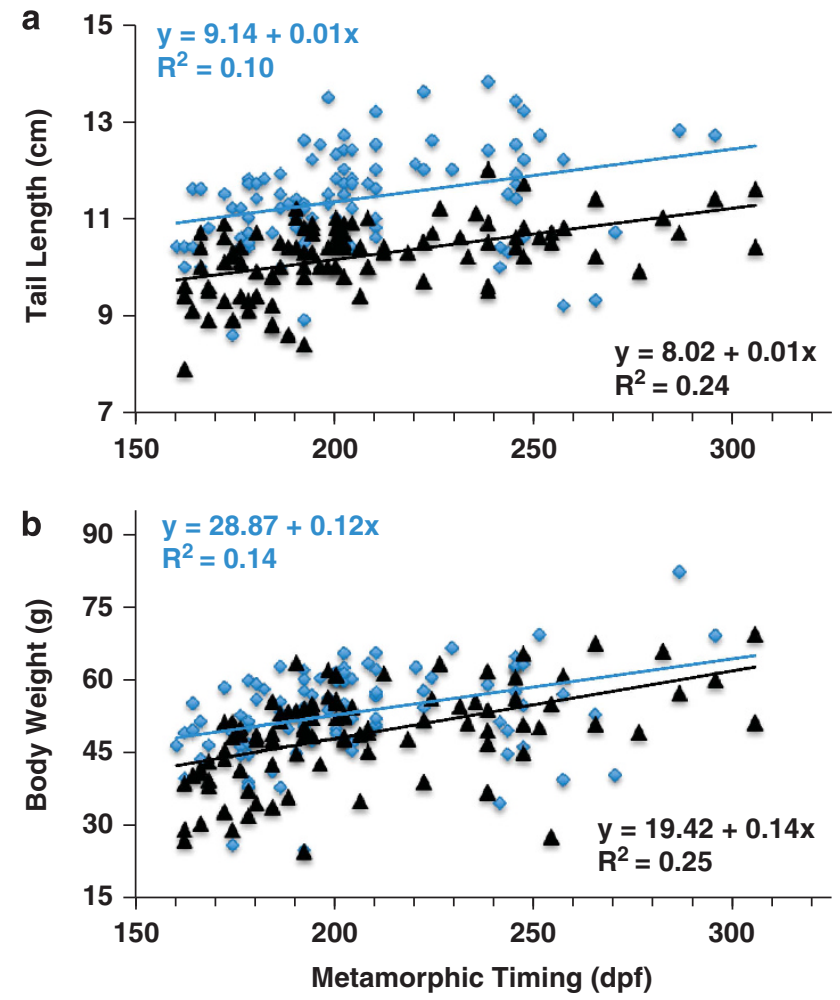

Figure 3 Plots showing the distribution of AxAn male (blue) and female (black) adult morphological traits relative to timing of metamorphosis. (a) Variation in tail length at $300 \mathrm{dpf}$. (b) Variation in body weight at $400 \mathrm{dpf}$. Best-fitting least squares regression lines are shown for each plot. Males presented significantly longer tails than females at $300 \mathrm{dpf}$ $\left(F_{(1,211)}=91.33, P<0.001\right)$ and were significantly heavier at $400 \mathrm{dpf}$ $\left(F_{(1,213)}=10.47, P=0.001\right)$.

Significant and maximal LOD scores for snout-vent length at $300 \mathrm{dpf}(\mathrm{LOD}=3.05)$, tail length at $300 \mathrm{dpf}(\mathrm{LOD}=3.30)$ and body weight at $400 \mathrm{dpf}(\mathrm{LOD}=2.76)$ were located to the maximum LOD inflection point of met2 (Figure 4); these were the only significant QTLs identified from whole-genome scans of the large Ambystoma 

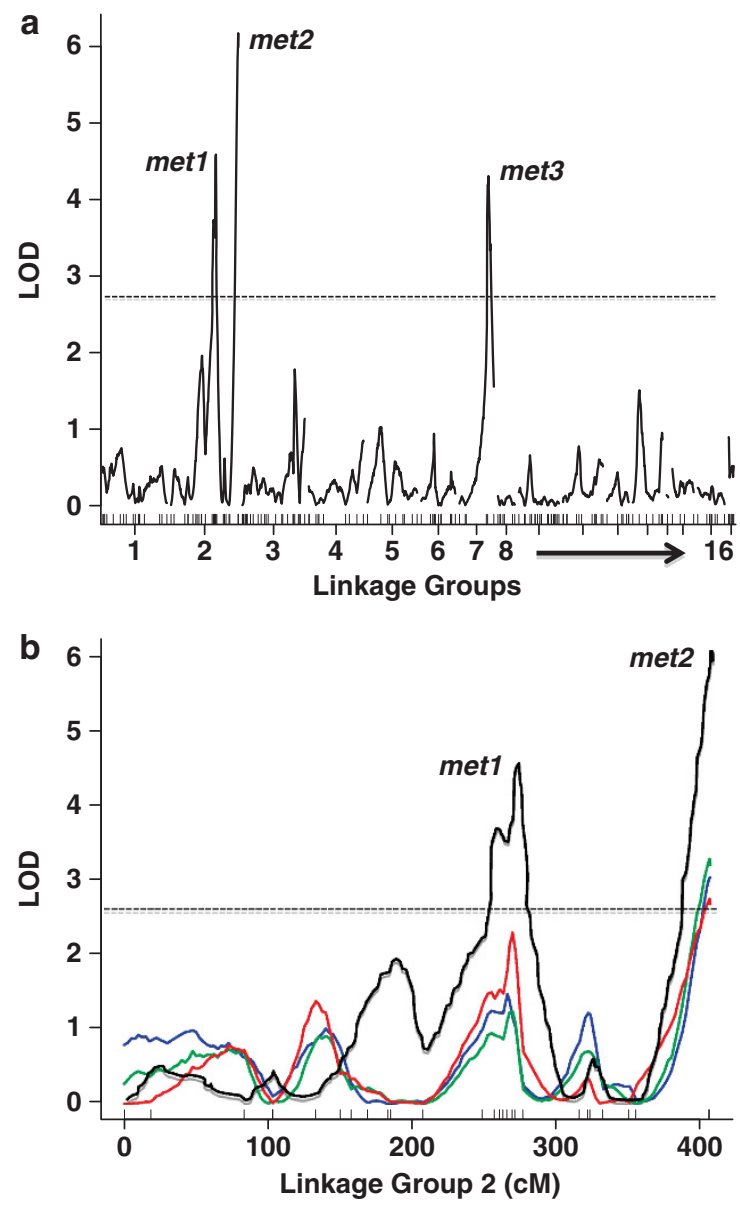

Figure 4 LOD plots of QTL scans. (a) Results of a genome-wide scan for metamorphic timing QTL; markers defining 16 ambystomatid linkage groups are concatenated on the $x$ axis. (b) Results of the genome-wide QTL scan for snout-vent length (blue), tail length (green) and body weight (red). The QTL metamorphic timing LOD plot is shown in black to reference the positions of met1 and met2. Horizontal dashed lines show the QTL significance threshold $(P=0.05)$.

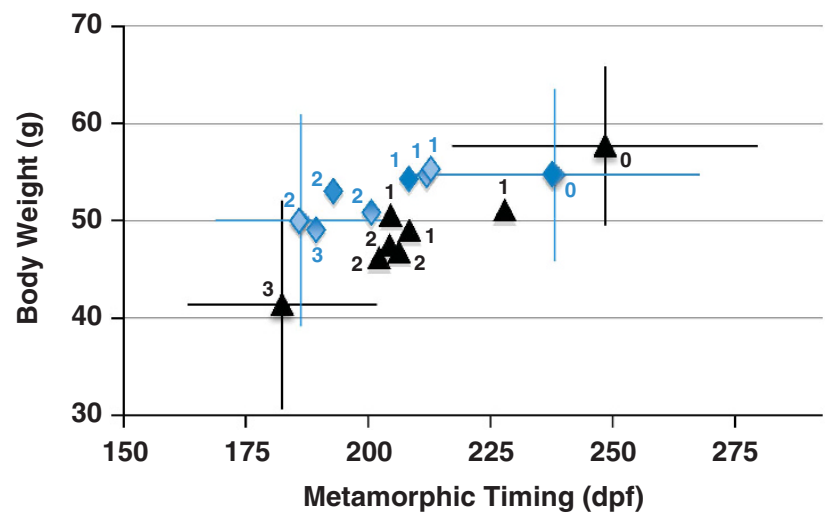

Figure 5 Plot showing mean body size and metamorphic timing for threelocus met1-3 genotypes. A total of eight genotypes are formed from the segregation of alleles among three unlinked loci in a backcross design. Male (blue) and female (black) means were plotted separately and numbers associated with symbols reference the number of $A$. andersoni alleles per genotype $(0-3)$. Error bars (s.d.) are only shown for the triple heterozygous and homozygous genotypes. genetic map, the largest known for any animal (Voss et al., 2011). The LOD profile for body weight mirrored the metamorphic timing profile at met1, and the LOD score was suggestive but not quite significant $(\mathrm{LOD}=2.42$, Figure 4$)$. Considerable among-individual variation was observed for morphological traits; however, the average effect of met genotypes was additive across loci. For example, metamorphic timing and body size varied positively as a function of the number of $A$. andersoni alleles that were inherited across met1-3 (Figure 5). On average, triply homozygous females with only A. mexicanum alleles at met1-3 metamorphosed 66 days later than triple heterozygotes $\left(\mathrm{F}_{(1,54)}=89.95, P<0.0001\right)$ and this significantly increased average adult body weight at $400 \mathrm{dpa}$ by $12 \mathrm{~g}$ or $38 \%$ $\left(\mathrm{F}_{(1,61)}=15.09, P<0.0003\right)$. Thus, QTL for adult morphological traits map to met2 and possibly met1, and met1-3 alleles additively explain variation in adult morphological traits.

\section{DISCUSSION}

Our study investigated the physiological and genetic basis of developmental timing variation in a classic example of evolutionary novelty-the evolution of paedomorphic salamanders (Gould, 1977). We showed that TH response varies among paedomorphic species and moderate effect TH-responsive QTL segregate allelic variation between $A$. andersoni and $A$. mexicanum. Individuals that inherited $A$. andersoni met $1-3$ alleles showed a more rapid response to $\mathrm{TH}$ on average, and as a result, an earlier time to complete metamorphosis. In turn, individuals that metamorphosed early presented smaller body sizes as adults. In contrast, individuals that inherited A. mexicanum met alleles delayed metamorphosis and attained significantly larger adult body sizes on average. Thus, our results show that substitution of alleles at TH-responsive loci explains variation arising from a classical life history trade-off, the dependency of adult body size variation on timing of metamorphosis.

It is well established that environmental factors affect metamorphic timing in amphibian populations and there is an important fitness relationship between metamorphic timing, survival and adult morphology (Wilbur and Collins, 1973; Gould, 1977; Wilbur, 1980; Werner, 1986; Semlitsch et al., 1988; Whiteman, 1994; Ryan and Semlitsch, 1998). Trade-offs in body size and metamorphic timing arise when metamorphosis is activated at variable times during development. For example, when some species of amphibian larvae are reared under sub-optimal conditions, growth and development are slowed and metamorphosis occurs early. If metamorphosis is activated early, a cost in adult body size is incurred because less time is spent in the rapid growth larval phase, and the energetic cost of metamorphosis may be inversely proportional to body size. When conditions are optimal, larvae metamorphose at larger body sizes, and such individuals reach reproductive maturity at earlier ages. Our results are the first to identify heritable genetic variation for a physiological mechanism-responsiveness to $\mathrm{TH}$ - that explains lifehistory trade-offs in an amphibian. Whether met1-3 regulates metamorphic timing variation in response to environmental cues is currently under investigation.

The LOD profiles for met2 and morphology QTL overlapped precisely, and these were the only significant morphology QTLs identified from whole-genome scans of the large Ambystoma genetic map, the largest known for any animal (Voss et al., 2011). This suggests that metamorphic timing and adult morphology QTL correspond to the same genes, supporting the idea (but not proving) that evolution of paedomorphosis is an example of adaptive pleiotropy. An alternative possibility is physical linkage of different genes. Although additional, 
independent crosses and larger sample sizes are needed to better establish met1 and met2 as QTLs for adult morphological traits, the results from this study firmly establish met1 as an ambystomatid metamorphic-timing QTL. In previous studies, met1 alleles from domesticated and wild-caught populations of A. mexicanum were shown to affect penetrance for paedomorph expression and delay metamorphic timing when evaluated in the genetic background of a metamorphic species (A. t. tigrinum; Voss and Shaffer, 2000; Voss and Smith, 2005). Voss and Smith (2005) found that homozygous $A$. mexicanum met1 genotypes delayed metamorphic timing by 36 days (on average), relative to heterozygous $A$. mexicanum/A. $t$. tigrinum genotypes. In the current study, homozygous A. mexicanum met 1 genotypes delayed metamorphic timing by 19 days (on average), relative to heterozygous $A$. mexicanum/A. andersoni genotypes. Thus, metamorphic and paedomorphic species carry met1 alleles that have varying effects on metamorphic timing. Presumably, if a backcross were performed in the A. andersoni direction, it would identify recessive alleles at different QTL than were identified in this study. This is because A. andersoni showed a more delayed response to $\mathrm{TH}$ than A. mexicanum (Figure 1). Our study suggests different genetic architectures for paedomorphosis in different tiger salamander lineages and, perhaps, standing genetic variation for metamorphic timing in natural populations (Voss and Shaffer, 1996; Voss et al., 2003).

All paedomorphic tiger salamanders that have been tested maintain capacity to undergo metamorphosis in response to T4. It is possible that metamorphosis remains operative because paedomorphic tiger salamander lineages are recently evolved and there has not been sufficient time for complete $\mathrm{TH}$ resistance to evolve (Marshall et al., 1994). Our study shows that in addition to the retention of metamorphosis, paedomorphs also retain a life-history trade-off associated with the ancestral, metamorphic life-history strategy. This vestige is likely retained because the same TH-responsive loci are used to regulate developmental timing variation in metamorphic and paedomorphic tiger salamanders. Consistent with endocrine control of metamorphosis and paedomorphosis (Darras and Kuhn, 1983; Galton, 1991; Rosenkilde and Ussing, 1996; Laudet, 2011), we hypothesize that these loci function centrally in the brain, where information is processed about maturational state, physiological condition, seasonality and environmental quality. TH levels are maintained at low levels during larval development by negative feedback regulation on the hypothalamus and pituitary. At metamorphosis, negative feedback regulation is lifted, $\mathrm{TH}$ levels increase and this further increases $\mathrm{TH}$ to critical levels that are required to induce peripheral metamorphic changes. Under this model, variation in metamorphic timing is expected to map to loci that regulate basal $\mathrm{TH}$ levels and/or alter the sensitivity to TH feedback.

If paedomorphic taxa generally arise by selection for delayed metamorphic timing, then these novel aquatic forms should generally present larger adult body sizes than related metamorphic forms. This hypothesis is supported by our results and also by phylogenetic studies (Wiens and Hoverman, 2008; Bonett et al., 2009). Indeed, paedomorphic salamanders are almost always much larger than terrestrial forms, with the paedomorphic Giant Chinese salamander (Andrias davidianis, total length $>1 \mathrm{~m}$ ) and US hellbender (Cryptobranchus alleganiensis, total length $>70 \mathrm{~cm}$ ) providing two extreme examples. These and other large paedomorphic species show extreme or complete insensitivity to TH (Laudet, 2011), thus supporting the idea that paedomorph evolution is associated with allele substitution at TH-responsive loci.

\section{DATA ARCHIVING}

QTL genotyping data are available via Sal-Site (at http://www. ambystoma.org/downloads/AxAn1genotypes.txt), a public website that archives DNA sequence data, microarray data and comparative mapping data available to the Ambystoma research community.

\section{CONFLICT OF INTEREST}

The authors declare no conflict of interest.

\section{ACKNOWLEDGEMENTS}

The data are available from SRV upon request. We thank Miranda Courtney for help with salamander care. This research was supported by grant 2R24OD010435 from the National Center for Research Resources (NCRR) and the Office of Research Infrastructure Programs (ORIP), a component of the National Institutes of Health (NIH). The project also used resources developed under Multidisciplinary University Research Initiative grant (W911NF-09-10305) from the Army Research Office (ARO), and National Science Foundation Grants IBN-9982719, IBN-0242833, IBN-0080112 and DBI0951484. GJ Voss was supported by a URAP fellowship from the ARO. The contents of this study are solely the responsibility of the authors and do not necessarily represent the official views of NCRR, NIH, ARO or NSF.

Bonett RM, Chippindale PT, Moler PE, Van Devender RW, Wake DB (2009). Evolution of gigantism in amphiumid salamanders. PLoS One 4: e5615.

Broman KW, Wu H, Sen S, Churchill GA (2003). R/qtl: QTL mapping in experimental crosses. Bioinformatics 19: 889-890.

Churchill GA, Doerge RW (1994). Empirical threshold values for quantitative trait mapping. Genetics 138: 963-971.

Darras VM, Kuhn ER (1983). Effects of TRH, bovine TSH, and pituitary extracts on thyroidal T4 release in Ambystoma mexicanum. Gen Comp Endocrinol 51: 286-291.

Duellman WE (1961). The amphibians and reptiles of Michoacán, México. Univ Kansas Pub Mus Nat Hist 15: 1-148.

Galton VA (1991). Thyroid hormone receptors and iodothyronine deiodinases in the developing Mexican axolotl, Ambystoma mexicanum. Gen Comp Endo 85: 62-70.

Gould SJ (1977). Ontogeny and Phylogeny. Belkap Press: Cambridge.

Howard RD, Moorman RS, Whiteman HH (1997). Differential effects of mate competition and mate choice on eastern tiger salamanders. Anim Behav 53: 1345-1356.

Kosambi D (1944). The estimation of map distances from recombination values. Ann Eugen 12: $172-175$.

Krenz JD, Sever DM (1995). Mating and ovoposition in paedomorphic Ambystoma talpoideum proceeds the arrival of terrestrial males. Herpetologica 51: 387-393.

Laudet V (2011). The origins and evolution of vertebrate metamorphosis. Curr Biol 21: R726-R737.

Marshall CR, Raff EC, Raff RA (1994). Dollo's law and the death and resurrection of genes. Proc Natl Acad Sci USA 91: 12283-12287.

Page RB, Voss SR, Samuels AK, Smith JJ, Putta S, Beachy CK (2008). Effect of thyroid hormone concentration on the transcriptional response underlying induced metamorphosis in the Mexican axolotl (Ambystoma). BMC Genomics 9: 78.

Rosenkilde P, Ussing AP (1996). What mechanisms control neoteny and regulate induced metamorphosis in urodeles? Int J Dev Biol 40: 665-673.

Ryan TJ, Semlitsch RD (1998). Intraspecific heterochrony and life history evolution: decoupling somatic and sexual development in a facultatively paedomorphic salamander. Proc NatI Acad Sci USA 95: 5643-5648

Semlitsch RD, Scott DE, Pechmann JHK (1988). Time and size at metamorphosis related to adult fitness in Ambystoma talpoideum. Ecology 69: 184-192.

Sen S, Churchill GA (2001). A statistical framework for quantitative trait mapping. Genetics 159: 37-387.

Shaffer HB (1984). Evolution in a paedomorphic lineage. I. An electrophoretic analysis of the Mexican ambystomatid salamanders. Evolution 38: 1194-1206.

Shaffer HB, McKnight ML (1996). The polytypic species revisited: genetic differentiation and molecular phylogenetics of the tiger salamander (Ambystoma tigrinum) (Amphibia: Caudata) complex. Evolution 50: 417-433.

Shaffer HB, Voss SR (1996). Phylogenetic and mechanistic analysis of a developmentally integrated character complex: alternate life history modes in ambystomatid salamanders. Am Zoo/ 36: 24-35.

Sprules WG (1974). The adaptive significance of paedogenesis in North American species of Ambystoma (Amphibia: Caudata): an hypothesis. Can J Zool 52: 393-400.

Voss SR, Shaffer HB (1996). What insights into the developmental traits of urodeles does the study of interspecific hybrids provide? Int J Dev Biol 40: 885-893.

Voss SR, Shaffer HB (1997). Adaptive evolution via a major gene effect: paedomorphosis in the Mexican axolotl. Proc Natl Acad Sci USA 94: 14185-14189.

Voss SR, Shaffer HB (2000). Evolutionary genetics of metamorphic failure using wild-caught versus laboratory axolotls (Ambystoma mexicanum). Mol Ecol 9: 1401-1408. 
Voss SR, Smith JJ (2005). Evolution of salamander life cycles: a major effect QTL contributes to both continuous and discrete variation for metamorphic timing. Genetics 170: 275-181.

Voss SR, Prudic K, Oliver J, Shaffer HB (2003). Candidate gene analysis of metamorphic timing in ambystomatid salamanders. Mol Ecol 12: 1217-1223.

Voss SR, Kump KD, Putta S, Pauly N, Reynolds A, Henry RJ et al. (2011). Origin of avian and amphibian chromosomes by fusion, fission, and retention of ancestral chromosomes. Genome Res 8: 1306-1312.
Weins JJ, Hoverman JT (2008). Digit reduction, body size, and paedomorphosis in salamanders. Evol Dev 10: 449-463.

Werner EE (1986). Amphibian metamorphosis: growth rate, predation risk and the optimum size at transformation. Am Nat 128: 319-341.

Whiteman HH (1994). Evolution of facultative paedomorphosis. Quart Rev Biol 69: 205-221. Wilbur HM (1980). Complex life cycles. Ann Rev Ecol Syst 11: 67-93.

Wilbur HM, Collins JP (1973). Ecological aspects of amphibian metamorphosis. Science 182: $1305-1314$.

Supplementary Information accompanies the paper on Heredity website (http://www.nature.com/hdy) 